\title{
Socio-Economic Profile of Sugarcane Growers in District Moradabad, Uttar Pradesh, India
}

\author{
Arun Kumar Pal*, Rahul Katiyar, H.C. Singh and Rajmani* \\ Department of Agricultural Extension, Chandra Shekhar Azad University of Agriculture and \\ Technology, Kanpur, Uttar Pradesh -208002, India \\ *Corresponding author
}

\section{A B S T R A C T}

The present study was conducted in Moradabad District of Western region of Uttar Pradesh. District Moradabad was selected purposively because the area under sugarcane cultivation is more in this district in comparison to other district of the four community

\begin{tabular}{|l|}
\hline Key w or d s \\
Sugarcane, Age, \\
Education, Family, \\
House. \\
\hline Article Info \\
\hline $\begin{array}{l}\text { Accepted: } \\
\text { 17 July } 2017 \\
\text { Available Online: } \\
\text { 10 September } 2017\end{array}$ \\
\hline
\end{tabular}
development blocks i.e. Chhajlet, Thakurdwara, Bilari and Moradabad taken randomly for drawing the sample of respondents. Five villages were selected for drawing sample from each block, from each village 10 sugarcane growers were selected on random basis. Total 200 respondents selected for the study. The Majority 75.00 per cent marginal 73.68 per cent small and 67.74 per cent medium farmers adopted agriculture as a main occupation. The Maximum sugarcane growers belonged to backward caste i.e. 58.06 per cent medium, 58.03 per cent marginal and 52.63 per cent small farmers. The Most of the 56.00 per cent respondents owned marginal (upto 1 ha) size of holding followed by 2.50 and 15.50 per cent respondents were having small and medium size of land holdings, respectively. The Most of the farmers i.e. 77.19 per cent small, 74.19 per cent medium and 37.50 per cent marginal farmers belonged to medium income group. The Most of 43.00 per cent sugarcane grower were using canal, 32.00 per cent canal + private tubewell /pump set as a source of irrigation. The Majority of 56.50 per cent farmers' sugarcane growers belonged to medium productively level where as 26.50 per cent belonged to low Productivity level and 17.00 per cent belonged to high productivity level.

\section{Introduction}

Agriculture is one of the most significant sectors of the Indian Economy. Agriculture is the only means of living for almost two thirds of the workers in India. The agriculture sector of India has occupied $43 \%$ of India's geographical area, and is contributing 13.9 per cent of India's GDP. Agriculture still contributes significantly to India's GDP despite decline of its share in India's GDP. There are number of crops grown by farmers. These include different food crops, commercial crops, oil seeds etc., sugarcane is one of the important commercial crops grown in India. Sugarcane is the main source of sugar in Asia and Europe. Sugarcane is grown primarily in the tropical and sub-tropical zones of the southern hemisphere. Sugarcane is the raw material for the production of white sugar, jaggery (gur) and khandsari. It is also used for chewing and extraction of juice for beverage purpose. The sugarcane cultivation and sugar industry in India plays a vital role 
towards socio-economic development in the rural areas by mobilizing rural resources and generating higher income and employment opportunities. About 7.5 percent of the rural population, covering about 45 million sugarcane farmers, their dependents and a large number of agricultural labour are involved in sugarcane cultivation, harvesting and ancillary activities. There are about nine States in India where sugarcane is grown on a large extent of area. There are a number of varieties that are grown in India depending on the suitability of the soil. The area, output and yield and sugarcane cultivation is subjected to fluctuate in response to policies of the government and also conditions of cultivation. Sugar factories have produced 18.91 million tonnes of sugar. With per capita consumption of white sugar at $22 \mathrm{~kg} /$ annum, and the estimated population of 1.50 billion by 2030 AD., the consumption of white sugar may be increased to 33 million tonnes. It is estimated that by 2030 AD., about 520 million tonnes of sugarcane with average sugar recovery of 10.75 per cent (60 per cent cane will be utilized for white sugar, and 15 per cent will go for ethanol production) will be required.

This will entail a productivity requirement of 100-110 tonnes/ha, as the area under sugarcane cultivation may stabilize around 5.00 million hectares. (Source: Indian Institute of Sugarcane research, Lucknow) Keeping in view the yield and recovery patterns of sugarcane during the last 25 years, the target is a stiff one, and it needs proper planning and judicious use of the available resources to achieve it. Intensive research efforts are, therefore, required to bring about this change in both cane quality and productivity. On an average, Uttar Pradesh commands 46 per cent of the cane acreage and 40 per cent sugarcane production. Thus, the onerous task of increasing the productivity rests squarely on Uttar Pradesh. In fact, the Indian Institute of Sugarcane Research based in Lucknow is working to this effect since its inception in 1952. The average cane yield of India has increased from 40.5 tonnes/ ha.(1950-51) to 70 tonnes/ha (2009-10), and recovery has gone up from 10.03 per cent to 10.20 per cent during this period, whereas in the same period, average yield of Uttar Pradesh has gone up from 39.48 tonnes/ha (1950-51) to 59.2 tonnes/ha (2009-10). (Source: Indian Institute of Sugarcane Research, Lucknow) Sugarcane is a moderately large sunny weather loving plant that is growing in two distinct climatic regions; the tropical and subtropical. The total area, under sugarcane in India is 44.08 lakh hectares, out of which $70 \%$ area lies in the subtropical belt (Uttar Pradesh, Haryana, Bihar and Punjab) and the remaining 30\% in the tropical region. In North India having subtropical climate, the important sugarcane growing states like Uttar Pradesh, Haryana, Bihar and Punjab. The average productivity of cane of the country is 68.84 tones/ ha and production is 338.96 million tones. (Source: Cooperative Sugar, March 2009, Vol-40, No.7).Uttar Pradesh, is the leading state of sugarcane growing and production than Haryana, Bihar, Punjab, Madhya Pradesh, Uttrakhand, etc.

The average production of Uttar Pradesh is 60.96 tones/ha which is lower than other state like Tamil Nadu, Karnataka, Maharashtra, Kerala etc. (tropical) and Punjab (subtropical). Thus it is urgently needed to increase the productivity of sugarcane for required level of supply to the factory one hand and prove its worth when compared with prevailing crops in the area on other hand.

\section{Materials and Methods}

The present study was conducted in Moradabad District of Western region of Uttar Pradesh. District Moradabad was selected purposively because the area under sugarcane cultivation is more in this district in 
comparison to other districts of the region and easily transportation facilities are available.

District Moradabad is comprises of 8 Blocks. In these all 8 community development blocks, farmers were cultivating popular and recommended sugarcane technology. The four community development blocks i.e. Chhajlet, Thakurdwara, Bilari and Moradabad taken randomly for drawing the sample of respondents. Chhajlet block is located on the Moradabad to Haridwar highway road. The Thakurdwara block is situated on the Moradabad to Kanshipur Road. The Bilari block is situated on the Moradabad to Chandausi road and Moradabad block is situated in Moradabad district.

\section{Percentage}

This was used for making simple comparison. To calculate the percentage, the frequency of the particular cell was multiplied by 100 and divided by the total number of respondents in that particular category. The formula used to calculate the percentage is given below.

Number of agreed respondents about a fact Percentage $=$--------------------- $\times 100$

Total number of respondents

\section{Mean score}

It was calculated to know the average value of a particular score. The formula used is as.

Total score on particular item

Mean score $=$

Number of respondent

This was used for each statement.

\section{Arithmetic mean}

The simplest and most important measure of average is arithmetic mean. It would be calculated with the help of the following formula-

Arithmetic mean $=\sum \mathrm{x} / \mathrm{n}$

Where,

$\sum=$ Sum

$\mathrm{x}=$ Variables

$\mathrm{n}=$ Number of observations

\section{Results and Discussion}

\section{Socio-economic profile of Sugarcane Growers}

\section{Age of sugarcane grower}

It is evident from table 1 that majority 52.68 per cent marginal farmers belonged to higher medium (46 to 60 years) of age group remaining 9.82, 24.11 and 13.39 per cent marginal farmers belonged to Young (18-30 years), Lower medium (31-45 years) and Old group (above 60 years) age group, respectively.

In case of small farmers maximum 50.88 per cent small farmers belonged to higher medium (46 to 60 years) of age group remaining 8.77, 28.07 and 12.28 per cent small farmers belonged to Young (18-30 years), Lower medium (31-45 years) and Old group (above 60 years) age group, respectively. The 48.39 per cent medium farmers belonged to higher medium (46 to 60 years) of age group remaining 12.90, 29.03 and 9.68 per cent medium farmers belonged to Young (15-30 years), Lower medium (3145 years) and Old group (above 60 years) age group, respectively.

Thus, it is clear from table 1 that most of the sugarcane growers belonged to higher medium (46 to 60 years) of age group that is 52.68 per cent marginal, 50.88 per cent small 
and 48.39 per cent medium farmers. It can be said that young farmers are very keen to grow sugarcane (Fig. 1).

\section{Education level}

It is apparent from table 2 that 24.11 per cent marginal farmers were having education up to primary followed by $21.43,17.86,16.07$, $9.82,3.57$ and 0.89 per cent marginal farmers were having up to intermediate, up to junior high school, high school, graduate, can read only and post graduate and above level of education, respectively.

Only 6.25 per cent marginal farmers were found illiterate. In case of small farmers 29.82 per cent were having up to primary level of education followed by $21.05,14.03,14.03$, $10.53,2.26$ and 1.75 per cent small farmers having up to high school, up to junior high school, intermediate, graduate, can read only and post graduate and above level of education, respectively. Only 3.51 per cent small farmers were found illiterate (Fig. 2).

In case of medium farmers 25.81 per cent were educated up to primary level of education followed by $22.58,16.13,12.90$, 6.45 and 6.45 per cent medium farmers having intermediate, graduate, up to high school, up to junior high school, can read only and post graduate and above level of education, respectively. None of the medium farmer was found in the category of illiterate.

Thus, it is clear from table 2 that maximum respondents were having up to primary level of education followed by intermediate, up to high school, up to junior high school, graduate, can read only and post graduate and above level of education. Only some sugarcane cultivators were found illiterate. It means most of the educated persons were having interest of sugarcane cultivation.

\section{Occupation of the sugarcane growers}

It is evident from table 3 that majority of the marginal 75.00 per cent, small 73.68 per cent and medium farmers 67.74 per cent were adopting agriculture as a main occupation whereas 25.81 per cent medium, 22.81 per cent small and 21.43 per cent marginal farmers were adopting agriculture + business, the 6.45 per cent medium, 3.57 per cent marginal and 3.51 per cent small farmers were doing agriculture + Business + service.

Thus, it is clear from table 3 that the majority (73.50 per cent) of the farmers were involved in agriculture, Only 22.50 per cent farmers were adopting Agriculture + Business and only 04.00 per cent farmers were adopting Agriculture + Business + Service (Fig. 3) .

\section{Caste composition}

It is evident from table 4 that the maximum 58.03 per cent marginal farmers belonged to backward caste followed by 21.43 per cent and 20.54 per cent belonging to general caste and scheduled caste, respectively (Fig. 4).

In case of small farmers maximum 52.63 per cent belonged to backward caste and 28.07 per cent belonged to general caste and 19.30 per cent belonged to scheduled caste. In case of medium farmers maximum 58.06 per cent belonged to backward caste followed by 35.49 and 6.4 per cent belonging to general caste and scheduled caste, respectively.

Thus, it is clear from table 4 that the most of the sugarcane growers belonged to backward caste.

It means backward people were using sugarcane as a cash crop, which will be helpful for upliftment of the socio-economic status of sugarcane growers. 
Distribution of sugarcane growers as per size of land holding

The table 5 revealed that 28.50 per cent respondents owned small size of holdings, while 56.00 per cent respondents had marginal size of holdings and only 15.50 per cent respondents had medium size of holdings. It is clear that the majority of the sugarcane growers belonged to marginal farmers category followed by small and medium farmers, respectively. It is evident from table 6 that majority 66.08 per cent marginal farmers were having medium (5 to 8 members) size of family (Fig. 5). Fallowed by 23.21 and 10.71 per cent were having small size of family (1 to 4 members) and large size of family (above 8 members), respectively. Whereas 64.91 per cent small farmers were having medium size of family (4 to 8 members) fallowed by 19.30 and 15.79 per cent were having small (1 to 4 members) and large (above 8 members) in family size, respectively. Similarity in case of medium farmers 61.30 per cent were having medium family (5 to 8 members) fallowed by 35.49 and 12.90 per cent were having small (1 to 4 members) and large (4 to 8 members) size of family, respectively.

Thus, it is clear from table 6 that the majority of the respondents were having medium family (4 to 8 members) who were growing sugarcane.

Table.1 Age of sugarcane growers ( $\mathrm{N}=200)$

\begin{tabular}{|l|l|l|l|l|l|l|}
\hline S.N. & Age level & $\begin{array}{l}\text { Marginal } \\
\mathbf{N = 1 1 2}\end{array}$ & $\begin{array}{l}\text { Small } \\
\mathbf{N = 5 7}\end{array}$ & $\begin{array}{l}\text { Medium } \\
\mathbf{N = 3 1}\end{array}$ & Total & Per cent \\
\hline $\mathbf{1 .}$ & $\begin{array}{l}\text { Young } \\
(18-30 \text { years })\end{array}$ & $\begin{array}{l}11 \\
(9.82)\end{array}$ & $\begin{array}{l}05 \\
(8.77)\end{array}$ & $\begin{array}{l}04 \\
(12.90)\end{array}$ & 20 & 10.00 \\
\hline $\mathbf{2 .}$ & $\begin{array}{l}\text { Lower medium (31- } \\
\text { 45 years) }\end{array}$ & $\begin{array}{l}27 \\
(24.11)\end{array}$ & $\begin{array}{l}16 \\
(28.07)\end{array}$ & $\begin{array}{l}09 \\
(29.03)\end{array}$ & 52 & 26.00 \\
\hline $\mathbf{3 .}$ & $\begin{array}{l}\text { Higher medium (46- } \\
\text { 60 years) }\end{array}$ & $\begin{array}{l}59 \\
(52.68)\end{array}$ & $\begin{array}{l}29 \\
(50.88)\end{array}$ & $\begin{array}{l}15 \\
(48.39)\end{array}$ & 103 & 51.50 \\
\hline $\mathbf{4 .}$ & $\begin{array}{l}\text { Old group (above 60 } \\
\text { year) }\end{array}$ & $\begin{array}{l}07 \\
(15.39)\end{array}$ & $\begin{array}{l}03 \\
(9.68)\end{array}$ & 25 & 12.50 \\
\hline
\end{tabular}

Table.2 Education level of sugarcane growers $(\mathrm{N}=200)$

\begin{tabular}{|l|l|l|l|l|l|l|}
\hline $\begin{array}{l}\text { S.N } \\
\boldsymbol{.}\end{array}$ & Education level & $\begin{array}{l}\text { Marginal } \\
\mathbf{N = ~ 1 1 2}\end{array}$ & $\begin{array}{l}\text { Small } \\
\mathbf{N = 5 7}\end{array}$ & $\begin{array}{l}\text { Medium } \\
\mathbf{N = 3 1}\end{array}$ & Total & Per cent \\
\hline $\mathbf{1 .}$ & Illiterate & $\begin{array}{l}07 \\
(6.25)\end{array}$ & $\begin{array}{l}2 \\
(3.51)\end{array}$ & - & 09 & 04.50 \\
\hline $\mathbf{2 .}$ & Can read only & $\begin{array}{l}04 \\
(3.57)\end{array}$ & $\begin{array}{l}3 \\
(5.26)\end{array}$ & $\begin{array}{l}02 \\
(6.45)\end{array}$ & 09 & 04.50 \\
\hline $\mathbf{3 .}$ & Up to primary & $\begin{array}{l}27 \\
(24.11)\end{array}$ & $\begin{array}{l}17 \\
(29.82)\end{array}$ & $\begin{array}{l}08 \\
(25.81)\end{array}$ & 52 & 26.00 \\
\hline $\mathbf{4 .}$ & Up to junior high school & $\begin{array}{l}20 \\
(17.86)\end{array}$ & $\begin{array}{l}08 \\
(14.03)\end{array}$ & $\begin{array}{l}03 \\
(9.68)\end{array}$ & 31 & 15.50 \\
\hline $\mathbf{5 .}$ & Up to high school & $\begin{array}{l}18 \\
(16.07)\end{array}$ & $\begin{array}{l}12 \\
(21.05)\end{array}$ & $\begin{array}{l}04 \\
(12.90)\end{array}$ & 34 & 17.00 \\
\hline $\mathbf{6 .}$ & Intermediate & $\begin{array}{l}24 \\
(21.43)\end{array}$ & $\begin{array}{l}08 \\
(14.03)\end{array}$ & $\begin{array}{l}07 \\
(22.58)\end{array}$ & 39 & 19.50 \\
\hline $\mathbf{7 .}$ & Graduate & $\begin{array}{l}06 \\
(9.82)\end{array}$ & $\begin{array}{l}06 \\
(10.53)\end{array}$ & $\begin{array}{l}05 \\
(16.13)\end{array}$ & 22 & 11.00 \\
\hline $\mathbf{8 .}$ & Post graduate and above & $\begin{array}{l}01 \\
(0.89)\end{array}$ & $\begin{array}{l}01 \\
(1.75)\end{array}$ & $\begin{array}{l}02 \\
(6.45)\end{array}$ & 04 & 02.00 \\
\hline
\end{tabular}


Table.3 Occupation of the sugarcane growers $(\mathrm{N}=200)$

\begin{tabular}{|c|c|c|c|c|c|c|}
\hline S.N. & Occupation level & $\begin{array}{l}\text { Marginal } \\
\mathrm{N}=112\end{array}$ & $\begin{array}{l}\text { Small } \\
\mathrm{N}=57\end{array}$ & $\begin{array}{l}\text { Medium } \\
\mathrm{N}=31\end{array}$ & Total & Per cent \\
\hline 1. & Agriculture & $\begin{array}{l}84 \\
(75.00)\end{array}$ & $\begin{array}{l}42 \\
(73.68)\end{array}$ & $\begin{array}{l}21 \\
(67.74)\end{array}$ & 147 & 73.50 \\
\hline 2. & $\begin{array}{l}\text { Agriculture+ } \\
\text { Business }\end{array}$ & $\begin{array}{l}24 \\
(21.43)\end{array}$ & $\begin{array}{l}13 \\
(22.81)\end{array}$ & $\begin{array}{l}08 \\
(25.81)\end{array}$ & 45 & 22.50 \\
\hline 3. & $\begin{array}{l}\text { Agriculture+ } \\
\text { Business+ service }\end{array}$ & $\begin{array}{l}04 \\
(3.57)\end{array}$ & $\begin{array}{l}02 \\
(3.51)\end{array}$ & $\begin{array}{l}02 \\
(6.45)\end{array}$ & 08 & 04.00 \\
\hline
\end{tabular}

Table.4 Caste composition of the sugarcane growers $(\mathrm{N}=200)$

\begin{tabular}{|l|l|l|l|l|l|l|}
\hline S.N. & Caste category & $\begin{array}{l}\text { Marginal } \\
\mathbf{N = ~ 1 1 2}\end{array}$ & $\begin{array}{l}\text { Small } \\
\mathbf{N = 5 7}\end{array}$ & $\begin{array}{l}\text { Medium } \\
\mathbf{N = 3 1}\end{array}$ & Total & Per cent \\
\hline 1. & Scheduled caste & $\begin{array}{l}23 \\
(20.54)\end{array}$ & $\begin{array}{l}11 \\
(19.30)\end{array}$ & $\begin{array}{l}02 \\
(6.45)\end{array}$ & 36 & 18.00 \\
\hline 2. & Backward caste & $\begin{array}{l}65 \\
(58.03)\end{array}$ & $\begin{array}{l}30 \\
(52.63)\end{array}$ & $\begin{array}{l}18 \\
(58.06)\end{array}$ & 113 & 56.50 \\
\hline 3. & General caste & $\begin{array}{l}24 \\
(21.43)\end{array}$ & $\begin{array}{l}16 \\
(28.07)\end{array}$ & $\begin{array}{l}11 \\
(35.49)\end{array}$ & 51 & 25.50 \\
\hline
\end{tabular}

Table.5 Distribution of sugarcane growers a per size of land holding ( $N=200)$

\begin{tabular}{|l|l|l|l|}
\hline S.N. & Particulars & Respondents \\
\cline { 3 - 4 } & & Number & Per cent \\
\hline 1. & Marginal farmers (up to 1 ha) & 112 & 56.00 \\
\hline 2. & Small farmers (1 to 2 ha) & 57 & 28.50 \\
\hline 3. & Medium farmers (2 to 4 ha) & 31 & 15.50 \\
\hline
\end{tabular}

Table.6 Family size of sugarcane growers $(\mathrm{N}=200)$

\begin{tabular}{|l|l|l|l|l|l|l|}
\hline S.N. & Particulars & $\begin{array}{l}\text { Marginal } \\
\mathbf{N = ~ 1 1 2}\end{array}$ & $\begin{array}{l}\text { Small } \\
\mathbf{N = 5 7}\end{array}$ & $\begin{array}{l}\text { Medium } \\
\mathbf{N = 3 1}\end{array}$ & Total & Per cent \\
\hline $\mathbf{1 .}$ & $\begin{array}{l}\text { Small } \\
(1 \text { to } 4 \text { members) }\end{array}$ & $\begin{array}{l}26 \\
(23.21)\end{array}$ & $\begin{array}{l}11 \\
(19.30)\end{array}$ & $\begin{array}{l}08 \\
(25.80)\end{array}$ & 45 & 22.50 \\
\hline $\mathbf{2 .}$ & $\begin{array}{l}\text { Medium } \\
\text { (5 to 8 members) }\end{array}$ & $\begin{array}{l}74 \\
(66.08)\end{array}$ & $\begin{array}{l}37 \\
(64.91)\end{array}$ & $\begin{array}{l}19 \\
(61.30)\end{array}$ & 130 & 65.00 \\
\hline 3. & $\begin{array}{l}\text { Large } \\
\text { (more than 8 members) }\end{array}$ & $\begin{array}{l}12 \\
(10.71)\end{array}$ & $\begin{array}{l}09 \\
(15.79\end{array}$ & $\begin{array}{l}04 \\
(12.90)\end{array}$ & 25 & 12.50 \\
\hline
\end{tabular}

Table.7 Family type of sugarcane growers $(\mathrm{N}=200)$

\begin{tabular}{|l|l|l|l|l|l|l|}
\hline S.N. & Particulars & $\begin{array}{l}\text { Marginal } \\
\mathbf{N = ~ 1 1 2}\end{array}$ & $\begin{array}{l}\text { Small } \\
\mathbf{N = \mathbf { 5 7 }}\end{array}$ & $\begin{array}{l}\text { Medium } \\
\mathbf{N = 3 1}\end{array}$ & Total & Per cent \\
\hline $\mathbf{1 .}$ & Nuclear family & $\begin{array}{l}45 \\
(40.18)\end{array}$ & $\begin{array}{l}17 \\
(29.82)\end{array}$ & $\begin{array}{l}09 \\
(29.03)\end{array}$ & 71 & 35.50 \\
\hline $\mathbf{2 .}$ & & $\begin{array}{l}67 \\
(59.82)\end{array}$ & $\begin{array}{l}40 \\
(70.18)\end{array}$ & $\begin{array}{l}22 \\
(70.97)\end{array}$ & 129 & 64.50 \\
\hline
\end{tabular}


Table.8 Annual income of sugarcane growers ( $\mathrm{N}=200)$

\begin{tabular}{|l|l|l|l|l|l|l|}
\hline $\begin{array}{l}\text { S. } \\
\text { N. }\end{array}$ & Annual income & $\begin{array}{l}\text { Marginal } \\
\mathbf{N = 1 1 2}\end{array}$ & $\begin{array}{l}\text { Small } \\
\mathbf{N = 5 7}\end{array}$ & $\begin{array}{l}\text { Medium } \\
\mathbf{N = 3 1}\end{array}$ & Total & Per cent \\
\hline $\mathbf{1 .}$ & $\begin{array}{l}\text { Lower income } \\
\text { (up to Rs. 15000) }\end{array}$ & $\begin{array}{l}54 \\
(48.21)\end{array}$ & $\begin{array}{l}03 \\
(5.26)\end{array}$ & $\begin{array}{l}01 \\
(3.22)\end{array}$ & 58 & 29.00 \\
\hline $\mathbf{2 .}$ & $\begin{array}{l}\text { Medium income (Rs. } \\
\text { 15001-50000) }\end{array}$ & $\begin{array}{l}42 \\
(37.50)\end{array}$ & $\begin{array}{l}44 \\
(77.19)\end{array}$ & $\begin{array}{l}23 \\
(74.19)\end{array}$ & 109 & 54.50 \\
\hline $\mathbf{3 .}$ & $\begin{array}{l}\text { Higher income } \\
\text { (Rs. Above 50000) }\end{array}$ & $\begin{array}{l}16 \\
(14.29)\end{array}$ & $\begin{array}{l}10 \\
(17.54)\end{array}$ & $\begin{array}{l}07 \\
(22.59)\end{array}$ & 33 & 16.5 \\
\hline
\end{tabular}

Table.9 Social participation of sugarcane growers $(\mathrm{N}=200)$

\begin{tabular}{|l|l|l|l|l|l|l|}
\hline S. N. & Particulars & $\begin{array}{l}\text { Marginal } \\
\mathbf{N = 1 1 2}\end{array}$ & $\begin{array}{l}\text { Small } \\
\mathbf{N = \mathbf { 5 7 }}\end{array}$ & $\begin{array}{l}\text { Medium } \\
\mathbf{N = 3 1}\end{array}$ & Total & Per cent \\
\hline $\mathbf{1 .}$ & $\begin{array}{l}\text { No membership of any } \\
\text { organization }\end{array}$ & $\begin{array}{l}09 \\
(8.04)\end{array}$ & $\begin{array}{l}03 \\
(5.26)\end{array}$ & - & 12 & 6.00 \\
\hline $\mathbf{2 .}$ & $\begin{array}{l}\text { Membership of one } \\
\text { organization }\end{array}$ & $\begin{array}{l}78 \\
(69.64)\end{array}$ & $\begin{array}{l}33 \\
(57.90)\end{array}$ & $\begin{array}{l}15 \\
(48.39)\end{array}$ & 126 & 63.00 \\
\hline $\mathbf{3 .}$ & $\begin{array}{l}\text { Membership of more } \\
\text { than one organization }\end{array}$ & $\begin{array}{l}16 \\
(14.29)\end{array}$ & $\begin{array}{l}14 \\
(24.56)\end{array}$ & $\begin{array}{l}11 \\
(35.48)\end{array}$ & 41 & 20.50 \\
\hline $\mathbf{4 .}$ & Office bearer & $\begin{array}{l}09 \\
(8.03)\end{array}$ & $\begin{array}{l}07 \\
(12.28)\end{array}$ & $\begin{array}{l}05 \\
(16.13)\end{array}$ & 21 & 10.50 \\
\hline
\end{tabular}

Table.10 Material possession of sugarcane growers $(\mathrm{N}=200)$

\begin{tabular}{|l|l|l|l|l|l|l|}
\hline S.N. & Particulars & $\begin{array}{l}\text { Marginal } \\
\mathbf{N = 1 1 2}\end{array}$ & $\begin{array}{l}\text { Small } \\
\mathbf{N = 5 7}\end{array}$ & $\begin{array}{l}\text { Medium } \\
\mathbf{N = 3 1}\end{array}$ & Total & Per cent \\
\hline $\mathbf{1 .}$ & Bullock Cart & $\begin{array}{l}56 \\
(50.00)\end{array}$ & $\begin{array}{l}41 \\
(71.93)\end{array}$ & $\begin{array}{l}17 \\
(54.83)\end{array}$ & 114 & 57.00 \\
\hline $\mathbf{2 .}$ & Motorcycle & $\begin{array}{l}47 \\
(41.96)\end{array}$ & $\begin{array}{l}42 \\
(73.68)\end{array}$ & $\begin{array}{l}26 \\
(83.87)\end{array}$ & 115 & 57.50 \\
\hline $\mathbf{3 .}$ & Chaff Cutter & $\begin{array}{l}99 \\
(88.39)\end{array}$ & $\begin{array}{l}57 \\
(100.0)\end{array}$ & $\begin{array}{l}29 \\
(93.54)\end{array}$ & 185 & 92.50 \\
\hline $\mathbf{4 .}$ & Pump set & $\begin{array}{l}37 \\
(33.04)\end{array}$ & $\begin{array}{l}22 \\
(38.60)\end{array}$ & $\begin{array}{l}25 \\
(80.65)\end{array}$ & 185 & 92.50 \\
\hline $\mathbf{5 .}$ & Tube bell & - & $\begin{array}{l}31 \\
(54.39)\end{array}$ & $\begin{array}{l}31 \\
(100.0)\end{array}$ & 62 & 31.00 \\
\hline $\mathbf{6 .}$ & Chair \& Furniture & $\begin{array}{l}33 \\
(29.46)\end{array}$ & $\begin{array}{l}40 \\
(70.18)\end{array}$ & $\begin{array}{l}26 \\
(83.87)\end{array}$ & 99 & 49.50 \\
\hline $\mathbf{7 .}$ & Cultivator & $\begin{array}{l}49 \\
(39.29)\end{array}$ & $\begin{array}{l}31 \\
(100.96)\end{array}$ & 124 & 62.00 \\
\hline $\mathbf{8 .}$ & Tractor & $\begin{array}{l}29 \\
(5.03)\end{array}$ & $\begin{array}{l}27 \\
(87.09)\end{array}$ & 65 & 32.50 \\
\hline $\mathbf{9 .}$ & Crasher & - & $\begin{array}{l}02 \\
(3.51)\end{array}$ & $\begin{array}{l}05 \\
(16.13)\end{array}$ & 07 & 3.50 \\
\hline $\mathbf{1 0 .}$ & Sprayer & - & $\begin{array}{l}10 \\
(17.54)\end{array}$ & $\begin{array}{l}29 \\
(93.55)\end{array}$ & 36 & 18.00 \\
\hline
\end{tabular}


Table.11 Source of irrigation of the sugarcane growers $(\mathrm{N}=200)$

\begin{tabular}{|l|l|l|l|l|l|l|}
\hline S. N. & $\begin{array}{l}\text { Source of } \\
\text { irrigation }\end{array}$ & $\begin{array}{l}\text { Marginal } \\
\mathbf{N = 1 1 2}\end{array}$ & $\begin{array}{l}\text { Small } \\
\mathbf{N = 5 7}\end{array}$ & $\begin{array}{l}\text { Medium } \\
\mathbf{N = 3 1}\end{array}$ & Total & Per cent \\
\hline $\mathbf{1 .}$ & $\begin{array}{l}\text { Own tube well// } \\
\text { pump set }\end{array}$ & $\begin{array}{l}20 \\
(7.14)\end{array}$ & $\begin{array}{l}22 \\
(35.09)\end{array}$ & 50 & 25.00 \\
\hline $\mathbf{2 .}$ & Canal & $\begin{array}{l}55 \\
(49.11)\end{array}$ & $\begin{array}{l}25 \\
(43.86)\end{array}$ & $\begin{array}{l}06 \\
(19.35)\end{array}$ & 86 & 43.00 \\
\hline $\mathbf{3 .}$ & $\begin{array}{l}\text { Canal + private } \\
\text { tube well/ pump set }\end{array}$ & $\begin{array}{l}49 \\
(43.75)\end{array}$ & $\begin{array}{l}12 \\
(21.05)\end{array}$ & $\begin{array}{l}03 \\
(9.68)\end{array}$ & 64 & 32.00 \\
\hline
\end{tabular}

Fig.1 Age of sugarcane growers ( $=200)$

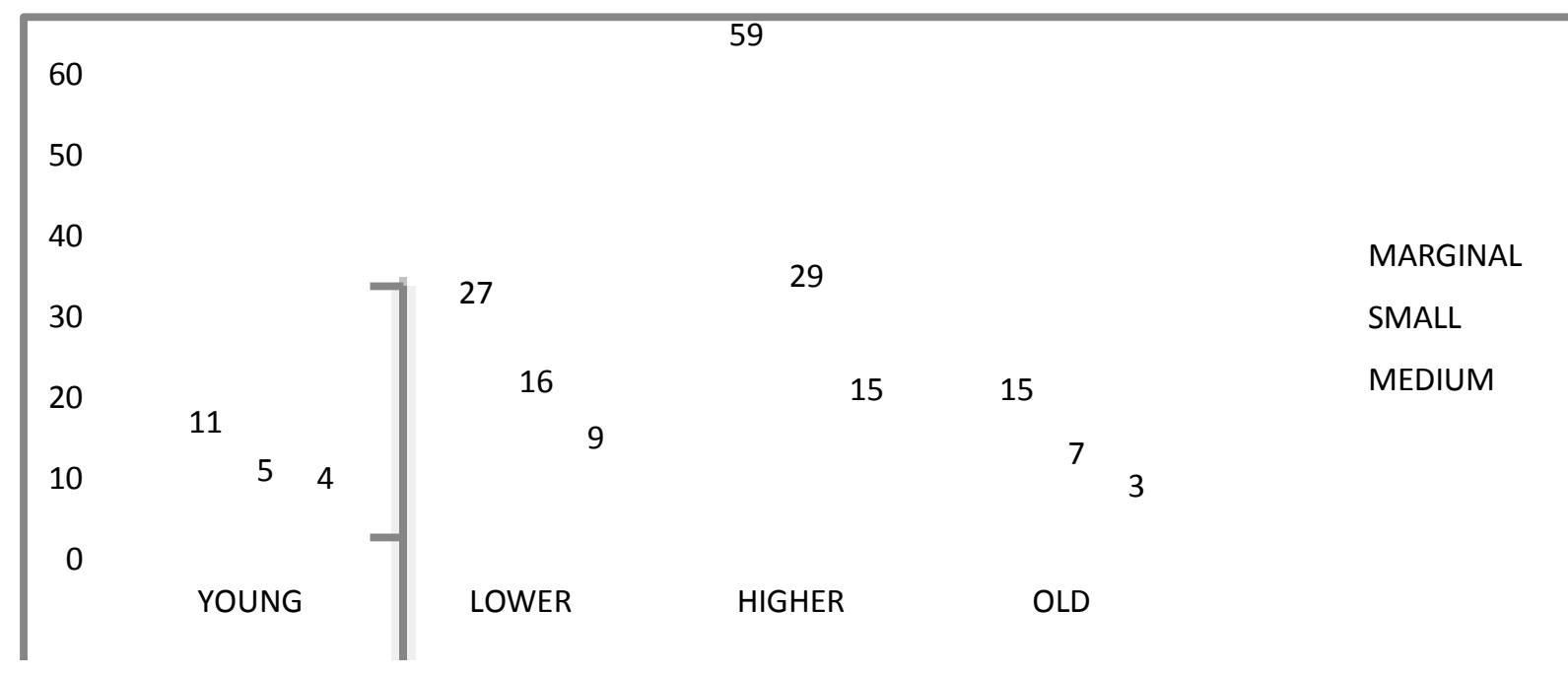

Fig.2 Education level of sugarcane growers (N=200)

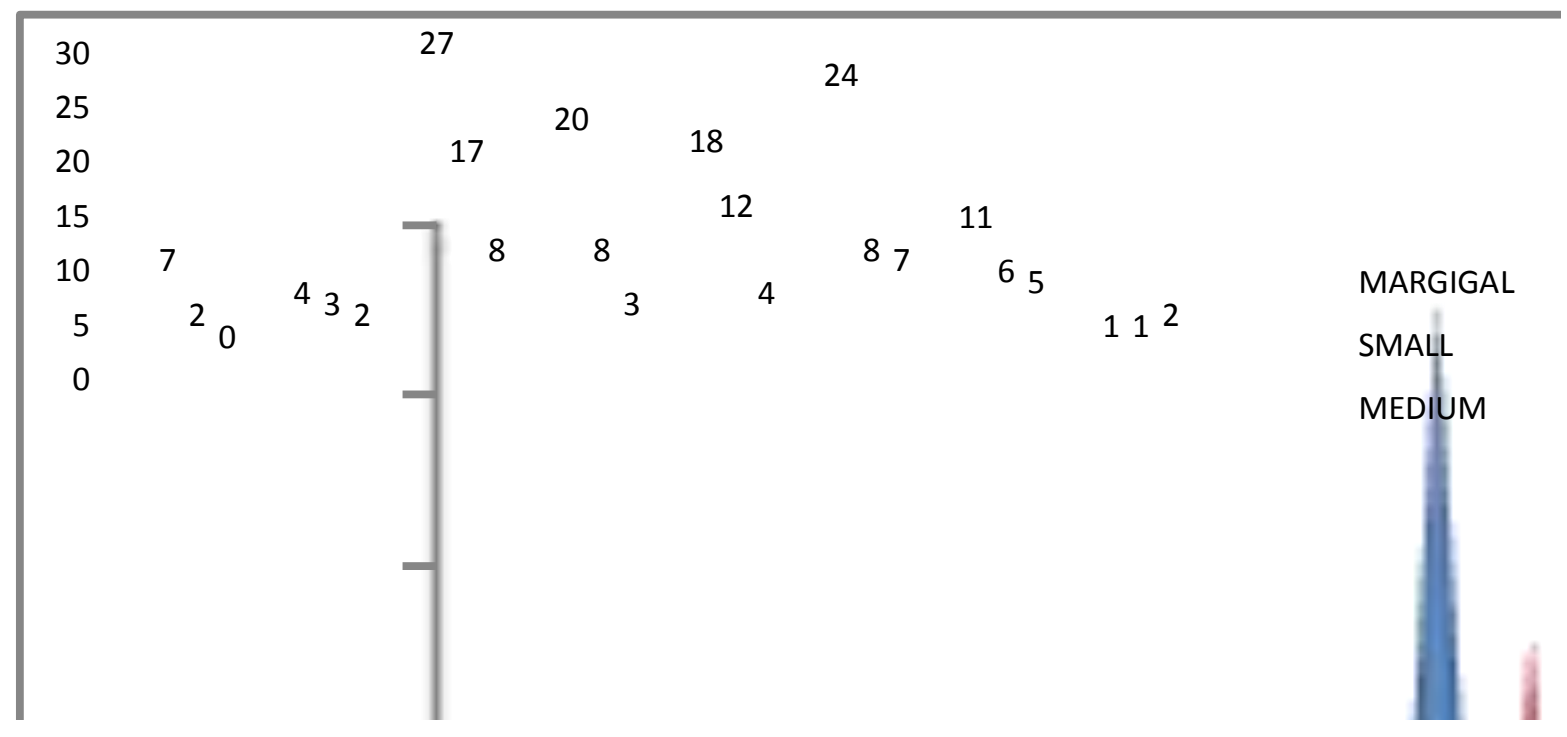


Fig.3 Occupation of the sugarcane growers $(\mathrm{N}=200)$

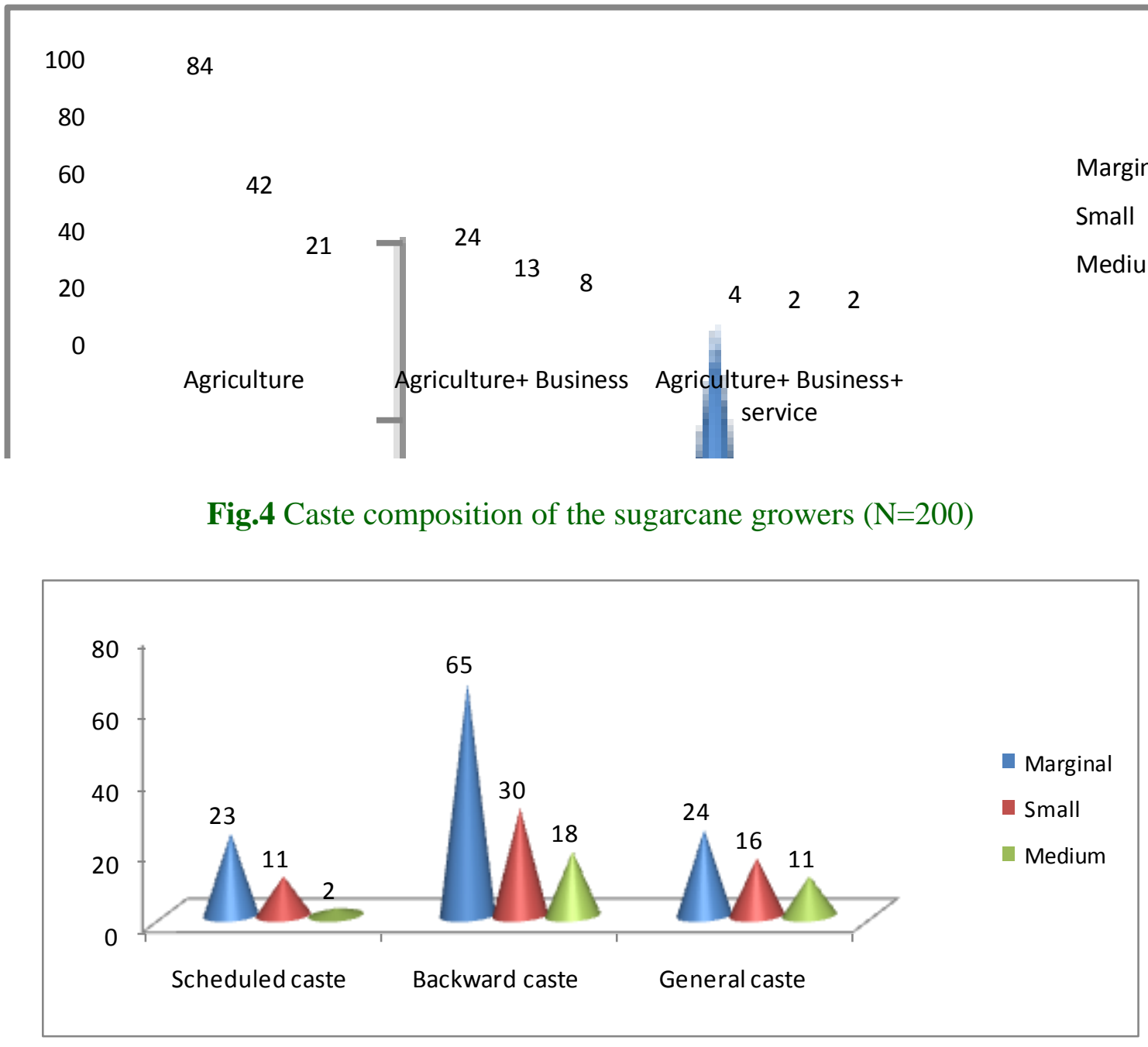

Fig.5 Family size of sugarcane growers

120

100

80

60

112

40

20

112

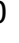

Marginal

Small

Medium

Fig.4 Caste composition of the sugarcane growers $(\mathrm{N}=200)$

Respondents
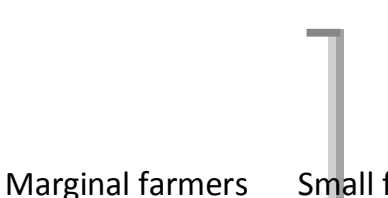

57

31

Small farmers Medium farmers 
Fig.6 Family type of sugarcane growers: $(\mathrm{N}=200)$

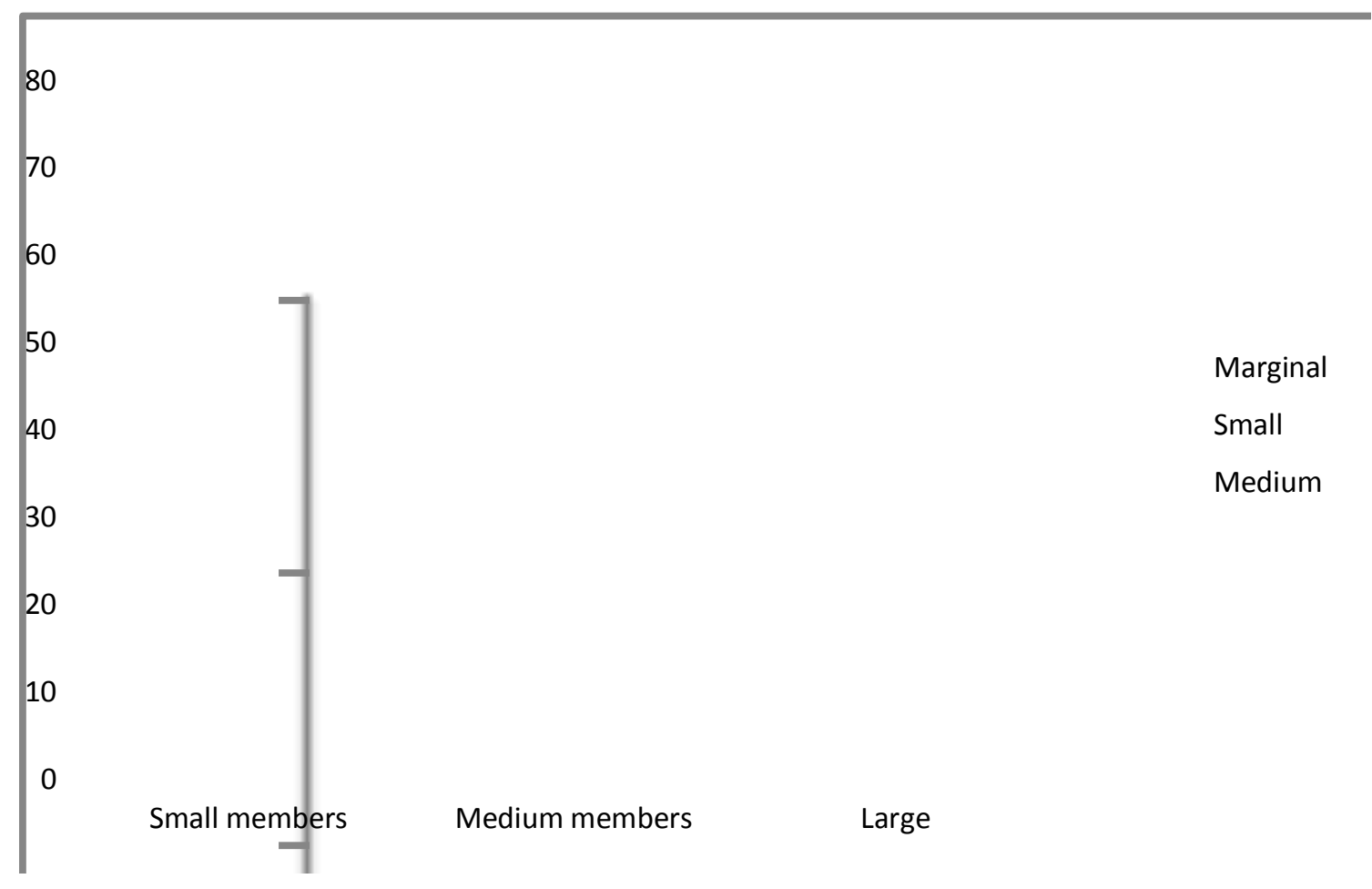

Fig.7 Annual income of sugarcane growers

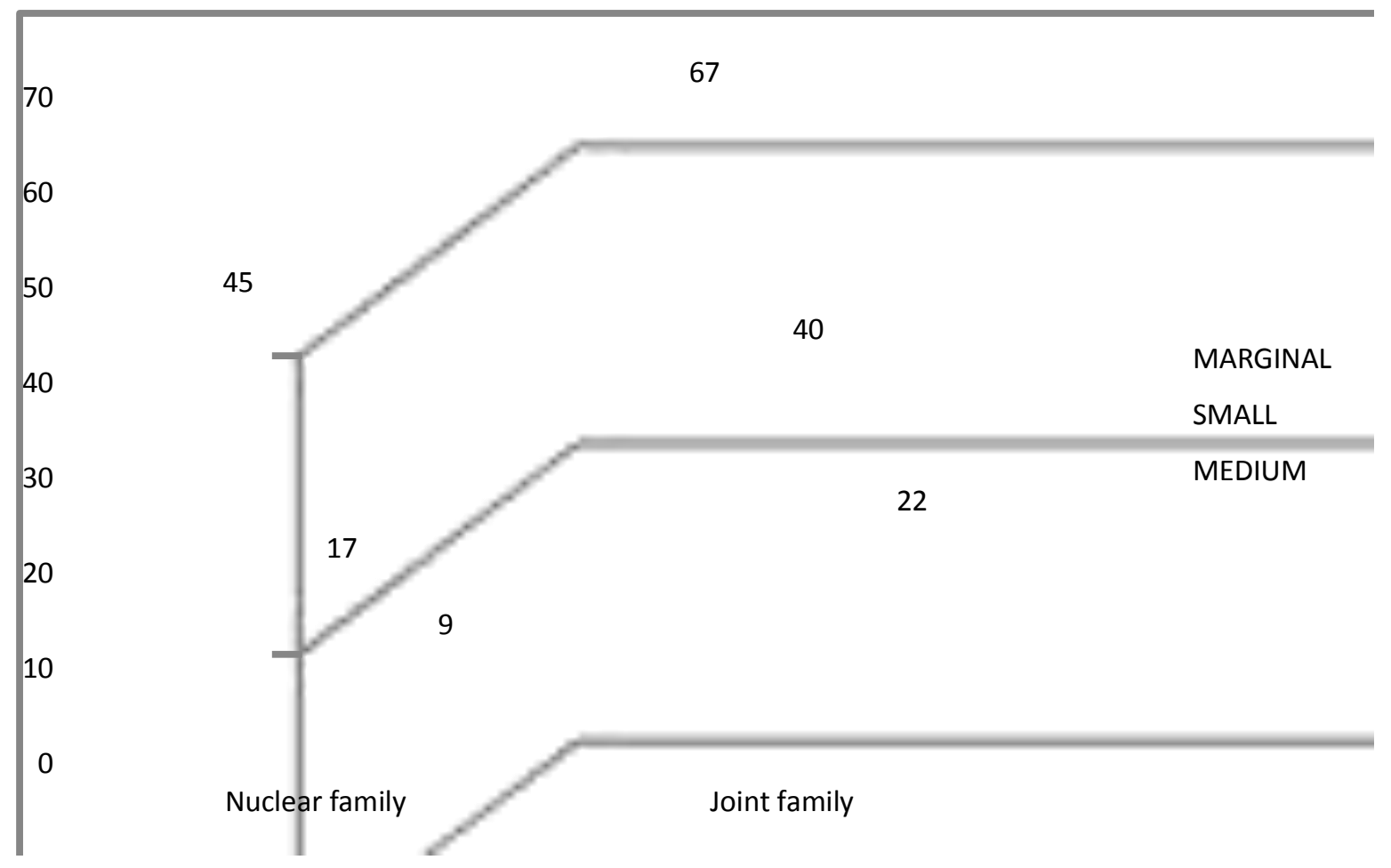


Fig.8 Social participation of sugarcane growers

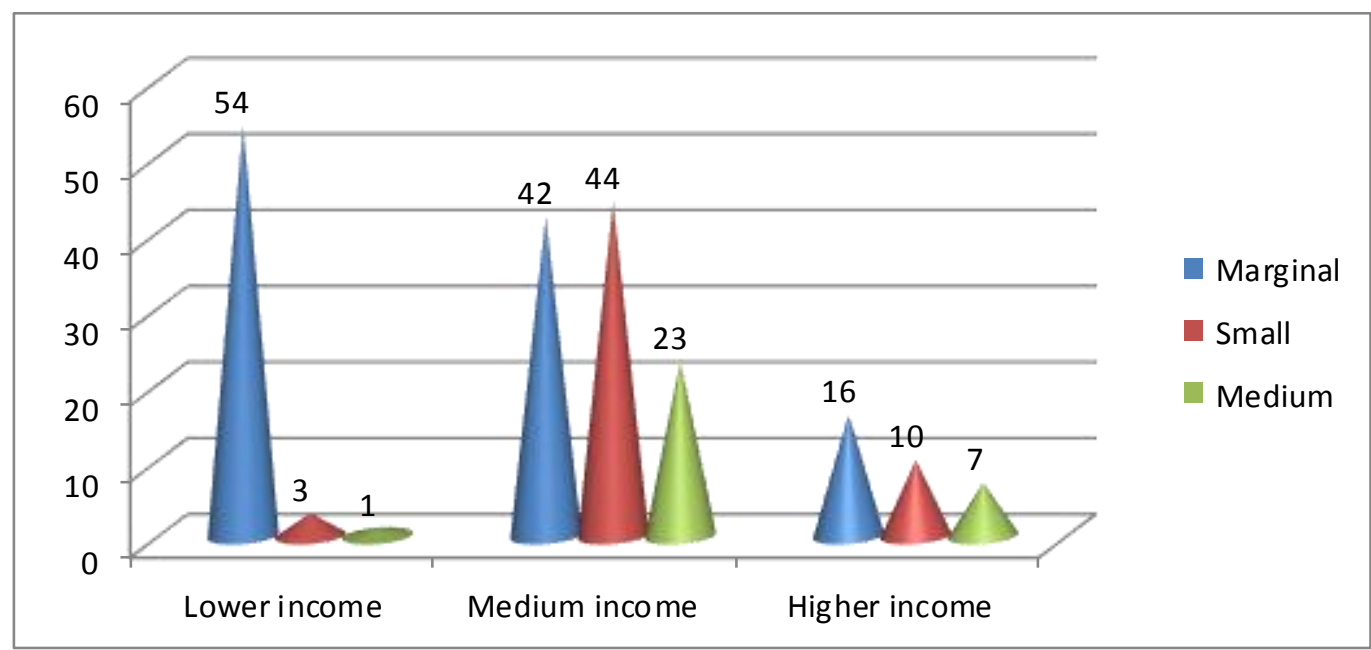

Fig.9 Social participation of sugarcane growers $(\mathrm{N}=200)$

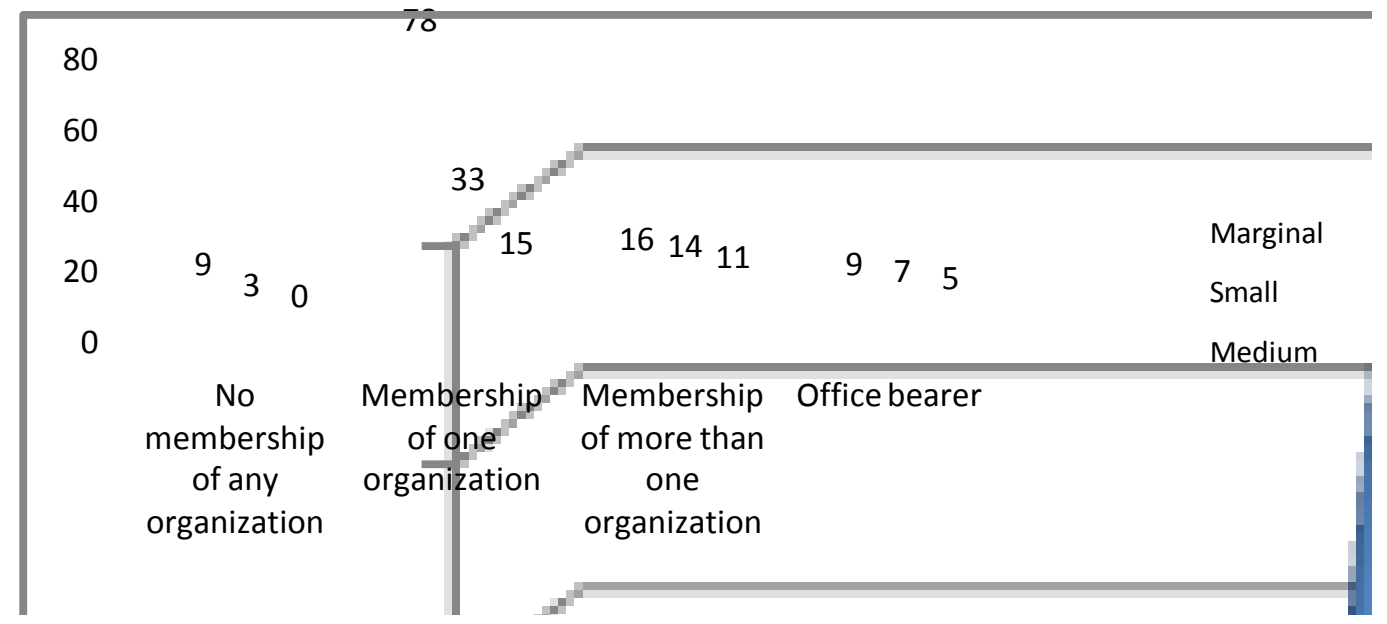

\section{Family type of sugarcane growers}

It is evident from table 7 that maximum 59.82 per cent marginal farmers were belonging to joint family system and 40.18 per cent were belonging to nuclear family system. Similarly 70.97 per cent medium farmers and 70.18 per cent small farmers belonged to joint family. The 40.18 per cent marginal farmers, 29.82 per cent small farmers and 29.03 per cent medium farmers, respectively belonged to nuclear family (Fig. 6).
Thus, it is clear from table 7 that most of the sugarcane growers belonged to joint family system.

It is evident from table 8 that maximum 48.21 per cent marginal farmers belonged to lower income group followed by 37.50 and 14.29 per cent marginal farmers belonging to medium and higher income groups, respectively. In case of small farmers majority 77.19 per cent belonged to medium income group followed by 17.5 per cent and 5.26 per cent belonging to high and low income 
groups, respectively. In case of medium farmers majority 74.19 per cent belonged to medium income group followed by 22.59 and 3.22 per cent belonging to high and low income groups, respectively.

Thus, it is clear from table 8 that the majority of the sugarcane growers belonged to medium income group and remaining belonged to either higher group or lower income group (Fig. 7).

It is revealed from table 9 that 69.64 per cent marginal farmers were having membership of one organization, 14.29 and 8.03 per cent marginal farmers were from membership of more than one organization and office bearer, respectively (Figs 8 and 9).

The 8.04 per cent marginal farmers were not having membership of any organization. In case of small farmers maximum 57.90 per cent were having membership of one organization followed by 24.56, 5.26 and 12.28 per cent were having membership of more than one organization, no membership if any organization and office bearer, respectively. In case of medium farmers maximum 48.39 per cent were having membership of one organization followed by 35.48 and 16.13 per cent medium farmers were having membership of more than one organization and office bearer, respectively.

In case of big farmers maximum 51.61 per cent were having membership of one organization followed by $38.70,9.67$ per cent big farmers were having membership of more than one organization and office bearer, respectively. There was no such Medium farmers reported who have no membership of any organization.

Thus, it is clear from table 9 that the most of the sugarcane growers were having either membership of the organization or membership of more than one organization.

\section{Material possession of sugarcane growers}

It is evident from table 10 that highest majority 88.39 per cent marginal farmers processed chaff cutter followed by 50.00 , $41.96,39.29,33.04,29.46$ ad 8.03 per cent marginal farmers who possessed bullock cart, motorcycle, cultivator, furniture and tractor, respectively. In case of small farmers highest majority 100.00 per cent possessed chaff cutter followed by 85.96, 73.68, 71.93, 70.18, $54.39,50.88,38.60,17.54$ and 3.51 per cent small farmers who possessed cultivator, Motorcycle, bullock cart, chair and furniture, tube well, tractor, pump set, sprayer and crasher, respectively. In case of medium farmers highest majority 100.0 and 100.0 per cent possessed cultivator and tube well followed by $93.55,93.54,87.09,83.87,83.87$, $80.65,54.83$, and 16.13 per cent possessed sprayer, tractor, motorcycle, chair and furniture, pump set, bullock cart and crasher, respectively.

Thus, it is clear from table 10 that medium farmers were having well of material possessions in comparison to the other categories.

\section{Source of irrigation of the sugarcane growers}

In case of medium farmers 70.97 per cent were using own tube well/ pump set as a source of irrigation fallowed by 9.68 and 19.35 per cent medium farmers were using canal + private tube well/pump set and canal as a source of irrigation.

On the basis of research findings the following conclusions were drawn that most of the farmers in agriculture belonged to backward casts, medium farmers' category, having above 5 to 8 members in a family, belonged to medium income group. In case of production level, most of the farmers belonged to the category of medium 
productively level. In case of adoption of sugarcane production technology, the highest gaps were found in intercropping, use of improved implements, seed treatments in all the categories of farmers. For the improvement of their technologies, the farmers need special training programme to provide sufficient information to improve and use properly.

Although, the gaps were found in significant manner in all practices which also needed to be improved for raising better crop. The correlation reveals between socio-economic status and adoption level of sugarcane production technology for all the practices in all the categories of farmers were positive and significant it means socio-economic status were having greater impact for the adoption of sugarcane technology in all areas. Lack of knowledge, not being aware about source of improved varieties, high cost of input, not aware about seed treatment, complexity of practices, high cost involved in method of planting, lack of planting skill, lack of proper irrigation facilities, scarcity of labours, lack of technical help, high cost of implements were the top most constraints as perceived by the all categories of sugarcane growers in adoption of sugarcane technology.

\section{References}

Gangwar, L.S., 2002. Problems and prospects of technology adoption in sugarcane production and Indian sugar industry. Indian Journal of Sugarcane Technology. 17 (1/2): 89-92.

Harsha Mendhe, and Jadhav, M.N. 2010. Significant relationship with the information seeking behavior of the sugarcane growers. Journal of Soils and Crops. 20(1): 118-122.

Hunshal, C.D., Khot, R.S. and Gaddnarker S.A. 1995. Row arrangement and fertilizer requirement of soya bean in sugarcane based intercropping system. Bharatiya Sugar., 21(7): 19-21.

Jaiswal, P.K., and Tiwari, R.K. 2014. Technological knowledge and adoption behavior of sugar cane growers of Surguja district, Chhattisgarh, South East Central India. Indian Journal Applied Research, 409.

\section{How to cite this article:}

Arun Kumar Pal, Rahul Katiyar, H.C. Singh and Rajmani. 2017. Socio-Economic Profile of Sugarcane Growers in District Moradabad, Uttar Pradesh. Int.J.Curr.Microbiol.App.Sci. 6(9): 1217-1229. doi: https://doi.org/10.20546/ijcmas.2017.609.148 